\title{
Predictive Value of Complete and Partial Early Virological Response on Sustained Virological Response Rates of Genotype-4 Chronic Hepatitis C Patients Treated with PEG-Interferon plus Ribavirin
}

\author{
I.S. Elefsiniotis E. Vezali C. Mihas G. Saroglou \\ University Department of Internal Medicine, Hepatology Unit, 'Elena Venizelou' Hospital, Athens, Greece
}

\section{Key Words}

Hepatitis C · Genotype $4 \cdot$ Early virological response •

PEG-interferon • Ribavirin

\begin{abstract}
Objectives: To investigate early viral kinetics, sustained virological response (SVR) rates and their predictors, in treatment-naïve, genotype-4-infected, chronic hepatitis C (CHC) patients treated with PEG-IFN $\alpha 2 \mathrm{~b}$ plus ribavirin. Patients and Methods: In total, 58 patients were retrospectively analyzed. Early virological response (EVR) was defined as undetectable HCV-RNA (<50 IU/ml) at week 12 (complete, cEVR) or at least a 2 log decrease in HCV-RNA levels (partial, pEVR). Results: Thirty-one patients exhibited SVR (53.4\%), 17 (29.3\%) were non-responders and $10(17.2 \%)$ relapsed. Thirty-seven patients (63.8\%) exhibited EVR. The positive predictive values of EVR, CEVR and pEVR for the SVR achievement were $83.87,54.83$, and $29.03 \%$, whereas their negative predictive values were $59.25,77.77$, and $81.48 \%$, respectively. Both cEVR (OR 0.040, $p=0.042$ ) and EVR (OR 0.016, $p=0.006)$ independently predicted SVR. Baseline viral load $(p<0.001)$, age $(p=$ $0.035)$ and stage of liver disease $(p=0.04)$ were significantly related to the EVR achievement, whereas only baseline viral load $(p=0.003)$ and ethnicity $(p=0.025)$ predicted cEVR. Conclusions: Partial or complete EVR represent independent predictors of SVR in genotype-4-infected CHC patients,
\end{abstract}

regardless of their baseline parameters. The absence of pEVR, rather than the absence of cEVR, should be used as an early indication for discontinuation of treatment in these patients.

Copyright $\odot 2009$ S. Karger AG, Basel

\section{Introduction}

The combination of interferon- $\alpha$ (IFN $\alpha$ ) and ribavirin (RIB) produced response rates in approximately $40 \%$ of previously untreated patients with chronic hepatitis $\mathrm{C}$ (CHC) $[1,2]$. Pegylated IFN $\alpha(\mathrm{PEG}-\mathrm{IFN} \alpha)$ plus RIB is currently the treatment of choice in patients with chronic hepatitis Cvirus (HCV)-related liver disease [3], achieving the highest rates of virological response observed to date, and treatment duration as well as RIB dose may be individualized by viral genotype [4]. Except for genotype, pretreatment viral load as well as patient age, body weight and liver histopathology (fibrosis/cirrhosis) are important predictors of response according to landmark trials [4-7].

Genotype 4 of HCV is the cause of approximately $20 \%$ of the 170 million global cases of CHC. Although rarely encountered in Western nations, it is the most common variant of HCV in Africa and the Middle East. In Egypt, the nation with the highest global prevalence of $\mathrm{HCV}$, 
more than $90 \%$ of cases are caused by genotype 4 . The genotype- 4 variant has also been detected among intravenous drug users (IVDUs) in Europe, predominantly in southern regions where incidence rates of up to $24 \%$ have been reported [8]. In Greece, the distribution of genotype- $4 \mathrm{HCV}$ infections among $\mathrm{CHC}$ cases is estimated between 13 and $15 \%$, according to the most recently published epidemiological studies $[9,10]$. Data concerning the efficacy of the currently approved combination treatment of genotype-4-infected CHC patients from Europe are limited. A retrospective analysis of genotype- $4 \mathrm{CHC}$ patients included in 2 studies, conducted primarily in Western countries $[4,6]$, suggests that sustained virological response (SVR) rates of $79 \%$ are attainable in patients with genotype-4 CHC receiving PEG-IFN $\alpha 2$ a plus RIB combination therapy for 48 weeks [11]. Unfortunately, the precise ethnic origins of the patients in this analysis are unknown, whereas there is a lack of data concerning genotype-4-infected CHC patients treated under real-life conditions (outside clinical trials) in Europe. Moreover, the influence of ethnicity on viral kinetics as well as on SVR rates of genotype-4-infected $\mathrm{CHC}$ patients treated with PEG-IFN $\alpha$ plus RIB is poorly investigated.

The principal aim of the present study was to investigate the early viral kinetics as well as the SVR rates and their predictors in treatment-naïve, genotype-4-infected $\mathrm{CHC}$ patients treated for 48 weeks with PEG-IFN $\alpha 2 \mathrm{~b}$ plus RIB under real-life conditions in Greece. The secondary aim of the study was to evaluate the predictive value of complete and/or partial early virological response (EVR) on the SVR rates of these patients.

\section{Methods}

Seventy-two consecutive, treatment-naïve patients with serologically, virologically and histologically confirmed genotype-4 CHC, with data available from medical records from 2002 to 2006, who had been treated with weight-adjusted doses of PEGIFN $\alpha 2 \mathrm{~b}$ and weight-adjusted RIB doses, were retrospectively analyzed. Inclusion criteria included: age between $\geq 18$ and $\leq 65$ years; detectable anti-HCV (ELISA III) at least 2 times within a year; detectable HCV-RNA in serum with a sensitive polymerase chain reaction (PCR) assay, qualitatively as well as quantitatively within a month before the beginning of treatment schedule; liver biopsy indicating chronic hepatitis within 6 months before treatment, and elevated alanine aminotransferase activity at start of treatment $(>40$ and $<400$ IU/l) and at least once during the last 6 months before the first visit.

Exclusion criteria included: decompensated liver disease as well as any other causes of chronic liver disease (chronic hepatitis $\mathrm{B} / \delta$, hemochromatosis, $\alpha_{1}$-antitrypsin deficiency, Wilson's disease, autoimmune hepatitis, alcohol-/drug-induced liver disease, etc.). Patients with HIV infection as well as active substance or alcohol abuse were also excluded. Laboratory values that excluded patients from treatment included: serum creatinine $>1.5 \mathrm{mg} / \mathrm{dl}$; absolute neutrophil count $<1,000 / \mu \mathrm{l}$; platelet count $<50,000 / \mu \mathrm{l}$, or hemoglobin $<10 \mathrm{~g} / \mathrm{dl}$ at baseline.

All patients were evaluated on admission clinically, hematologically, biochemically and serologically as well as with ultrasound of the upper abdomen. Routine biochemical and hematological tests were performed using automated techniques. Serological markers for viral hepatitis were detected using routine commercially available enzyme immunoassays $\left(\mathrm{AxSYM}{ }^{\circledR}, \mathrm{Ab}\right.$ bott Laboratories, Abbott Park, Ill., USA). Serum HCV-RNA levels were measured qualitatively (lower detection limit of $50 \mathrm{IU} /$ $\mathrm{ml}$ ) and quantitatively by a commercially available PCR assay (Cobas Amplicor HCV test, version 2, Roche Diagnostics, Branchburg, N.J., USA). HCV genotype was performed using INNOLIPA HCV assay (Innogenetics, Ghent, Belgium).

Liver biopsy was performed at baseline in all CHC patients according to Menghini technique, and all biopsy specimens were evaluated by a hepatopathologist and were scored according to the Ishak scoring system (grade 0-18, stage 0-6) [12].

All patients were treated with weight-adjusted doses of PEGIFN $\alpha 2 \mathrm{~b}$ (Peg-Intron, $1.5 \mu \mathrm{g} / \mathrm{kg} /$ week) and weight-adjusted RIB doses (Rebetol 1,000-1,200 mg/day, depending on the baseline body weight $<$ or $\geq 75 \mathrm{~kg}$, respectively). The duration of treatment was 48 weeks. All patients were clinically, hematologically and biochemically evaluated at 4, 8, 12, 24, 48 and 72 weeks of treatment/follow-up.

Serum HCV-RNA data were available before the initiation and at week 12 of treatment (qualitative and quantitative PCR assay) as well as at week 48 of treatment and 24 weeks after the end of the treatment schedule (qualitative PCR assay). An HCV-RNA of $>600,000 \mathrm{IU} / \mathrm{ml}$ was considered high (high viral load). An EVR was confirmed either by undetectable $(<50 \mathrm{IU} / \mathrm{ml})$ serum HCVRNA at week 12 (complete, cEVR) or by at least a 2 log decrease in HCV-RNA levels from baseline (partial, pEVR) at week 12 of treatment. The end of treatment virological response (EOT) was confirmed by undetectable serum HCV-RNA at week 48 , whereas a SVR was further confirmed by undetectable serum HCVRNA 24 weeks after completion of treatment. Patients with EOT but detectable serum HCV-RNA 24 weeks after completion of treatment were characterized as relapsers (RLs), whereas patients without EOT were characterized as non-responders (NRs) in the analyses. Patients who did not complete 48 weeks of treatment were excluded from the final analysis.

\section{Statistical Analysis}

Numerical characteristic values were tested for normality with the Shapiro-Wilk test. All continuous variables are presented as means \pm standard deviations or medians \pm interquartile ranges (75th-25th percentile) in case they deviated from normality. Categorical variables are shown as absolute and relative (percentages) frequencies. Contingency tables with calculation of Pearson's $\chi^{2}$ or Fisher's exact tests evaluated associations between the categorical variables. Depending on the distribution, the Mann-Whitney U test (for non-normal variables) or the Student's $t$ test were used for the comparisons of continuous variables between 2 groups. The Kruskal-Wallis statistic was calculated to compare the distribution of non-normal variables among 3 groups. Multiple logistic regression analysis was applied in order 
Table 1. Baseline (clinical, virological, biochemical and histological) data of the study population (Caucasians vs. Egyptians)

\begin{tabular}{lcc}
\hline & Caucasians $(\mathrm{n}=38)$ & Egyptians $(\mathrm{n}=34)$ \\
\hline Age, years & $42.97 \pm 10.10$ & $38.26 \pm 10.51^{*}$ \\
Gender (male/female) & $28 / 10$ & $26 / 8$ \\
BMI & $24.20 \pm 2.68$ & $26.03 \pm 2.98^{*}$ \\
HCV-RNA, IU/ml & $1,127,294 \pm 773,675$ & $1,328,547 \pm 637,780$ \\
HVL,\% & 65.8 & 82.3 \\
ALT, IU/l & $72.44 \pm 25.71$ & $93.00 \pm 45.54^{*}$ \\
AST, IU/1 & $44.72 \pm 16.78$ & $57.82 \pm 25.17^{*}$ \\
Grade (0-18) & $6.19 \pm 1.92$ & $5.65 \pm 1.42$ \\
Stage (0-6) & $2.41 \pm 1.47$ & $2.19 \pm 0.70$ \\
Cirrhosis (yes/no) & $5 / 33$ & $2 / 32$ \\
\hline
\end{tabular}

Data regarding continuous variables are presented as mean \pm standard deviation. $\mathrm{BMI}=$ Body mass index; $\mathrm{HVL}=$ high viral load $(>600,000 \mathrm{IU} / \mathrm{ml}) .{ }^{*} \mathrm{p}<0.05$.

Table 2. Early virological response (EVR) rates among patients with or without a sustained virological response (SVR)

\begin{tabular}{lrc}
\hline & SVR $(\mathrm{n}=31)$ & No SVR $(\mathrm{n}=27)$ \\
\hline EVR & $26 / 31(83.87 \%)$ & $11 / 27(40.75 \%)$ \\
cEVR & $17 / 31(54.83 \%)$ & $6 / 27(22.22 \%)$ \\
pEVR & $9 / 31(29.03 \%)$ & $5 / 27(18.52 \%)$ \\
\hline
\end{tabular}

$\mathrm{cEVR}=$ Complete early virological response; $\mathrm{pEVR}=$ partial early virological response.

to evaluate to what extent various characteristics are associated with SVR. Deviance residuals were calculated in order to evaluate the model's goodness-of-fit. All reported probability values ( $p$ values) were based on two-sided tests and compared to a significance level of 0.05 after Bonferroni adjustment for multiple comparisons. Stata ${ }^{\mathrm{TM}} 9.0$ statistical software was used for the statistical analysis (STATA Corp., College Station, Tex., USA).

\section{Results}

Fifty-four patients were men (75\%) and 18 women (25\%), whereas 38 (52.8\%) patients were Caucasians and 34 (47.2\%) were Egyptians. The majority of Caucasian patients $(34 / 38,89.47 \%)$ were prior IVDUs, whereas none of 34 Egyptian patients was a prior IVDU. Seven patients (9.7\%) had histologically confirmed cirrhosis and 53/72 (73.6\%) exhibited a high viral load at baseline control.
Baseline data among Caucasian and Egyptian patients are presented in table 1. Egyptian patients were younger and exhibited significantly higher BMI and baseline ALT/ AST levels compared to Caucasians. All the other baseline characteristics were comparable among these groups of patients.

Fifty-eight of 72 patients $(80.6 \%)$ were included in the final analysis. Fourteen patients (19.4\%), 8 Caucasians and 6 Egyptians, did not complete 48 weeks of treatment for various reasons. Overall, 31 patients exhibited SVR (53.4\%), 17 (29.3\%) were characterized as NRs, and 10 (17.2\%) were characterized as RLs. Fourteen Caucasian patients $(14 / 30,46.66 \%)$ and 17 Egyptian patients (17/28, $60.71 \%)$ exhibited SVR, whereas 9 Caucasian patients $(9 / 30,30 \%)$ and $8(8 / 28,28.57 \%)$ Egyptian patients were NRs and 4 Caucasian patients $(4 / 30,13.33 \%)$ and $6(6 / 28$, $21.42 \%)$ Egyptian patients were RLs. The response type was not significantly related to ethnicity $(\mathrm{p}=0.903)$ as well as the other baseline parameters, except for the presence of cirrhosis $(\mathrm{p}=0.028)$.

Thirty-seven patients (63.8\%) exhibited EVR (14 pEVR and $23 \mathrm{cEVR}$ ). Baseline viral load ( $<<0.001)$, patient age $(\mathrm{p}=0.035)$ and stage of liver disease $(\mathrm{p}=0.04)$ were significantly related to the achievement of EVR, whereas only baseline viral load ( $\mathrm{p}=0.003)$ and ethnicity $(\mathrm{p}=$ $0.025)$ were significantly related to the achievement of cEVR. In the multivariate model that was adjusted for all the baseline parameters studied, both cEVR (OR 0.040, 95\% CI 0.002-0.884, $\mathrm{p}=0.042$ ) and EVR (OR 0.016, 95\% CI $0.001-0.305, \mathrm{p}=0.006$ ) independently predicted SVR. According to the results of the analysis, as shown in table 2, the positive predictive values of EVR, cEVR and pEVR for the achievement of SVR were 83.87, 54.83, and $29.03 \%$, whereas their negative predictive values were $59.25,77.77$, and $81.48 \%$, respectively.

\section{Discussion}

Traditionally, the vast majority of genotype-4 $\mathrm{CHC}$ cases are observed in Egypt and in countries of Central and West Africa. Recently, HCV-4 has become increasingly prevalent in southern European countries of the Mediterranean basin, including Greece, especially in IVDUs and $\mathrm{HIV} / \mathrm{HCV}$ co-infected patients, mainly due to the movement of IVDUs across European borders and the active immigration of individuals from regions endemic for HCV-4 [8].

In the present study, the viral kinetic profiles and the SVR rates of a group of Caucasian and Egyptian geno- 
type-4-infected $\mathrm{CHC}$ patients, treated under real-life conditions in Greece, were analyzed. The main finding is that, despite some differences in their viral kinetic profile, Egyptian and Caucasian patients exhibit comparable SVR rates (60.71 vs. $46.66 \%)$, although they are lower than those reported from clinical trials $(65-69 \%)$ conducted in Egypt, Saudi Arabia, Qatar and Kuwait [13-15]. On the other hand, studies from Europe have shown that the SVR rates in African or European HCV-4 patients are lower than those achieved in studies conducted in the Middle East $[11,16]$, results similar to ours. An overall better response has been observed in Egyptian HCV-4 patients compared to European patients (54.9 vs. $40.3 \%$ ), but it remains unclear if this difference in SVR rates is related to ethnicity or other factors such as HCV-4 subtype, the mode of HCV transmission or the severity of liver disease. The 4 a subtype is largely predominant (93\%) in Egyptian patients, whereas the main route of transmission in European patients is IVDU [16]. Moreover, liver fibrosis is significantly less severe in European HCV-4 patients according to the results from European studies [16].

The 2 groups of the current study population were comparable for all the baseline parameters that could have an impact on the type of response (viral load, liver histology), except for age and BMI. The possible explanation is that all patients were treated with weight-adjusted doses of PEG-IFN $\alpha 2 b$ and RIB. Weight-adjusted therapies possibly have an advantage over fixed dosing as indicated by studies concluding that response rates are lower in overweight chronic HCV-infected patients [17]. Additionally, as shown in table 1 , the cohort did not include obese patients (mean BMI 24.2 and 26.03 in the Caucasian and Egyptian subgroups).

An EVR, defined either as undetectable HCV-RNA at week 12 or at least a 2 log decrease in HCV-RNA levels from baseline, was an excellent predictor of treatment outcome, whereas the absence of EVR was associated with virtually no chance of achieving SVR, according to the results from Egyptian studies [15, 18]. The present study evaluated the prognostic significance of 2 types of EVR (complete and partial EVR) for the SVR achievement. According to the logistic regression analysis, both pEVR and cEVR were independent predictors of SVR. The best positive predictive value (83.87\%) was achieved when both cEVR and pEVR were estimated together, whereas the best negative predictive value was that obtained from the estimation of pEVR (81.48\%). It is suggested that the absence of pEVR, rather than the absence of cEVR, should be used as an early indication for stopping treatment in these patients.

Egyptian patients exhibited significantly lower cEVR rates compared to Caucasians, as well as patients with a high baseline viral load $(>600,000 \mathrm{IU} / \mathrm{ml})$ compared to those with a low viral load. Using cEVR as an early indication for stopping the treatment schedule, a significant proportion of patients $(14 / 31,45.16 \%)$ who finally responded, especially from the Egyptian group, would have been lost. The probable explanations are the genetic-host factors and the genotype- 4 subtype-dependent differences, parameters which need further investigation.

Limitations of the study are its retrospective-observational nature and the absence of data concerning HCV-4 subtypes. However, the observational nature of this study adds a pragmatic flavor to 'real-life data', an aspect often missing from clinical trials. This study provides preliminary observations that need to be verified or falsified in large-scale randomized clinical trials.

In conclusion, SVR rates are comparable among Egyptian and Caucasian HCV-4 patients, treated under reallife conditions with the currently approved treatment, despite some differences in their early on-treatment virological data. Either pEVR or cEVR represent independent predictors of SVR in the majority of genotype-4-infected $\mathrm{CHC}$ patients, regardless of all the other baseline parameters. The absence of pEVR, rather than the absence of cEVR, should be used as an early indication for discontinuation of treatment in these patients.

\section{References}

1 McHutchinson JG, Gordon SC, Schiff ER, et al: Interferon alpha-2b alone or in combination with ribavirin as initial treatment for chronic hepatitis C. Hepatitis Interventional Therapy Group. N Engl J Med 1998;339: 1485-1492.

-2 Lai MY, Kao JH, Yang PM, et al: Long-term efficacy of ribavirin plus interferon alpha in the treatment of chronic hepatitis C. Gastroenterology 1996;111:1307-1312.
3 NIH Consensus Statement on Management of Hepatitis C: 2002. NIH Consens State Sci Statements 2002;19:1-46.

-4 Hadziyannis SJ, Sette H Jr, Morgan TR, et al: Peginterferon- $\alpha 2 \mathrm{a}$ and ribavirin combination therapy in chronic hepatitis C: a randomized study of treatment duration and ribavirin dose. Ann Intern Med 2004;140: 346-355. 
5 Manns MP, McHutchinson JG, Gordon SC, et al: Peginterferon alpha-2b plus ribavirin compared with interferon alpha-2b plus ribavirin for initial treatment of chronic hepatitis C: a randomized trial. Lancet 2001;358; 958-965.

-6 Fried MW, Shiffman ML, Reddy KR, et al: Peginterferon alpha-2a plus ribavirin for chronic hepatitis $\mathrm{C}$ virus infection. $\mathrm{N}$ Engl J Med 2002;347:975-982.

7 Heathcote EJ, Shiffman ML, Cooksley GE, et al: Peginterferon alpha-2a in patients with chronic hepatitis $\mathrm{C}$ and cirrhosis. N Engl J Med 2000;343:1673-1680.

$\checkmark 8$ Kamal SM, Nasser IA: Hepatitis C genotype 4: what we know and what we don't yet know. Hepatology 2008;47:1371-1383.

-9 Katsoulidou A, Sypsa V, Tassopoulos NC, et al: Molecular epidemiology of $\mathrm{HCV}$ in Greece: temporal trends in HCV genotypespecific incidence and molecular characterization of genotype 4 isolates. J Viral Hepat 2006;13:19-27.
10 Savvas S, Koskinas J, Sinani C, et al: Changes in epidemiological patterns of $\mathrm{HCV}$ infection and their impact on liver disease over the last 20 years in Greece. J Viral Hepat 2005; 12:551-557.

11 Diago M, Hassanein T, Rodés J, et al: Optimized virologic response in hepatitis $\mathrm{C}$ virus genotype 4 with peginterferon- $\alpha 2 \mathrm{a}$ and ribavirin. Ann Intern Med 2004;140:72-73.

12 Ishak K, Baptista A, Bianchi L, et al: Histological grading and staging of chronic hepatitis. J Hepatol 1995;22:696-699.

13 Kamal SM, El Kamary SS, Shardell MD, et al: Pegylated interferon- $\alpha 2 b$ plus ribavirin in patients with genotype 4 chronic hepatitis C: the role of rapid and early virological response. Hepatology 2007;46:1732-1740.

14 Hasan F, Asker H, Al-Khaldi J, et al: Peginterferon alpha-2b plus ribavirin for the treatment of chronic hepatitis $\mathrm{C}$ genotype 4 . Am J Gastroenterol 2004;99:1733-1737.
15 Kamal SM, El Tawil AA, Nakano T, et al: Peginterferon alpha-2b and ribavirin therapy in chronic hepatitis C genotype 4: impact of treatment duration and viral kinetics on sustained virological response. Gut 2005;54: 858-866.

16 Roulot D, Bourcier V, Grando V, et al; Observational VHC4 Study Group: Epidemiological characteristics and response to peginterferon plus ribavirin treatment of hepatitis $\mathrm{C}$ virus genotype 4 infection. J Viral Hepat 2007; 14:460-467.

17 Tarantino G, Conca P, Sorrentino P, Ariello M: Metabolic factors involved in the therapeutic response of patients with hepatitis $\mathrm{C}$ virus-related chronic hepatitis. J Gastroenterol Hepatol 2006;21:1266-1268.

18 El-Zayadi AR, Attia M, Barakat EM, et al: Response of HCV genotype 4 naïve patients to 24 weeks of PEG-IFNa2b/ribavirin or induction dose IFNa2b/ribavirin/amantadine: a non-randomized controlled study. Am J Gastroenterol 2005;100:2447-2452. 